\title{
Anchored Instruction: Situiertes Lernen in multimedialen Lernumgebungen
}

\section{Ursula Scharnhorst}

"Anchored Instruction" ist ein konstruktivistischer Ansatz aus den USA, dessen Vertreter technologisch unterstützte, situierte Lernumgebungen für verschiedene schulische Fächer und Altersstufen entwickeln, implementieren und erforschen. Wichtige Ziele, Gestaltungsprinzipien und Merkmale solcher Lernumgebungen sowie ihre Implementation im Unterricht werden anhand von zwei Anwendungen näher dargestellt: (1) "The Adventures of Jasper Woodbury", eine Serie von Video-Geschichten und Zusatzmaterialien, die Schüler mit komplexen mathematischen Problemstellungen konfrontieren, und (2) "The Little Planet Literacy Series", die Schulanfänger mit Trickfilm-Geschichten und damit verbundenen multimedialen Lernaktivitäten ins Lesen und Schreiben einfïhren. Es wird auch kurz auf einige Forschungsergebnisse zu den beiden Lernumgebungen verwiesen. Im Weiteren verdeutlicht eine Taxonomie, dass soziale und technologische Formen der Lernunterstützung bei "Anchored Instruction" vielfältig kombiniert werden, um die Flexibiliät und den Transfer des Gelernten zu fördern. Im Hinblick auf die aufwändige Erstellung und Implementation solcher Lernumgebungen wird am Schluss eine Reihe kritischer Fragen und Einwände diskutiert.

Theoretischer Hintergrund und allgemeine Ziele von Anchored Instruction

$\mathrm{Zu}$ Beginn wird der Anchored Instruction Ansatz in seinen grösseren theoretischen Bezugsrahmen gestellt. Die wichtigsten allgemeinen Zielsetzungen und Annahmen, die damit verbunden sind, sollen in den folgenden Abschnitten kurz angesprochen werden.

Anchored Instruction als konstruktivistischer Ansatz Anchored Instruction ${ }^{1}$ ist ein pädagogisch-psychologischer Ansatz, der am Learning Technology Center ${ }^{2}$ (LTC) in Vanderbilt entwickelt wurde. Was den theoretischen Hintergrund betrifft, beziehen sich die Vertreter von Anchored In- 
struction auf neuere konstruktivistische Auffassungen des Lernens, zu deren Formulierung und konkreter Umsetzung sie viel beigetragen haben (z.B. CTGV, 1990, 1993a, 1993b, 1997). Ausführliche Darstellungen der Grundannahmen und der verschiedenen Ausprägungen des Konstruktivismus finden sich bei Duffy \& Jonassen (1992) und bei Gerstenmeier \& Mandl (1995). Anchored Instruction versucht, zentrale Forderungen der Vertreter der situierten Kognition bzw. des situierten Lernens umzusetzen (Brown, Collins \& Duguid,1989; Greeno, Smith \& Moore, 1993; Lave \& Wenger, 1991; Resnick, 1987; Rogoff, 1990). Dazu gehört, dass Wissen nicht einfach passiv vermittelt werden kann, sondern von den Lernenden in der aktiven Auseinandersetzung mit Lernsituationen konstruiert wird. Lernprozesse und Lernprodukte in Form von deklarativem und prozeduralen Wissen sind daher eng mit den jeweiligen Lernsituationen verknüpft. Lernen wird grundsätzlich als situations- und kontextgebunden verstanden, d.h. Vorwissen und individuelle Lernermerkmale interagieren mit Merkmalen der Lernsituation.

Bei Anchored Instruction wird auf passive und dekontextualisierte Wissensvermittlung verzichtet. Das Ziel ist vielmehr, technologisch unterstützte Lernumgebungen zu konzipieren, in denen aktives, problemorientiertes Lernen gefordert und gefördert wird. Sinn und Zweck der Lernhandlungen und des Gelernten sollen deutlich werden, indem die Lernenden authentische ${ }^{3}$, fallbezogene Problemstellungen bearbeiten, die den Einbezug von Vorwissen und Alltagsdenken ermöglichen (CTGV, 1992a). Flexible Nutzung, Generalisierung und Transfer des erworbenen Wissens auf andere Situationen bleiben dennoch wichtige Ziele. Umgebungen, die nach den Prinzipien von Anchored Instruction gestaltet sind, ermöglichen den Lernenden - ausgehend von Ankerproblemen - erworbenes Wissen und Fertigkeiten auf andere, zunehmend unterschiedliche Lern- und Problemlösesituationen $\mathrm{zu}$ übertragen und so die flexible Anwendung zu sichern (CTGV, 1990, S. 6).

In neueren konstruktivistischen Theorien werden Lernen, Denken und Problemlösen zudem als sozial geteilte Aktivitäten betrachtet, die sozio-kulturell geprägt sind und als soziale Praktiken vermittelt werden (z.B. Lave, 1991; Resnick, 1987). In Anchored Instruction Umgebungen wird deshalb viel Wert auf kollaboratives Lernen und Problemlösen in Peergruppen gelegt, in denen sich Schüler gegenseitig unterstützen können (CTGV, 1993a, 134). Die authentischen und komplexen Problemstellungen sollen auch ermöglichen, dass vermehrt Formen der Unterstützung in den Unterricht einfliessen, die typischerweise in «apprenticeships» (Brown, Collins \& Duguid, 1989) zwischen Experten und Novizen praktiziert werden (CTGV, 1990, 1992b). Dazu gehören sowohl das Anregen explorativer Lern- und Problemlöseprozesse als auch die Demonstration und das Coaching durch Lehrpersonen oder kompetente Peers, die dazu beitragen, dass wichtige Aspekte der Problemlösung artikuliert und reflektiert werden. Die Lernumgebungen bieten jedoch nicht nur einen gemeinsamen Lern- und Problemlösekontext für Lehrpersonen, Schüler und weitere Bezugs- 
personen, sondern sie werden auch im Hinblick auf ihre teamorientierte, multidisziplinäre Entwicklung und Erforschung als Kontexte verstanden, die Kollaboration, verteilte Expertise und kollektives Verstehen in «learning communities» (Brown und Campione, 1994) begünstigen (CTGV, 1997, 119-121).

Gemeinsam mit andern konstruktivistischen Ansätzen (Brown \& Campione, 1994; Scardamaglia, Bereiter \& Lamon, 1994) bildet Anchored Instruction auch die theoretische und praktische Grundlage für breit angelegte reformpädagogische Forschungsprojekte in den USA, die unter dem Begriff «Schools for Thought» bekannt sind (Lamonet. al. 1996).

\section{Träges Wissen}

Die Notwendigkeit situierter Lernumgebungen wird vor allem damit begründet, dass unter traditionellen Instruktionsbedingungen erworbenes Wissen oft träge bleibt (Bransford, Franks, Vye \& Sherwood, 1989; Mandl, Gruber, \& Renkl, 1993; Scardamaglia \& Bereiter, 1985; Sherwood, Kinzer, Hasselbring \& Bransford, 1987). Der Begriff des trägen Wissens wurde schon früh geprägt (Whitehead, 1929). Er drückt aus, dass Wissensbestände über faktenorientierte, verbale Instruktion zwar gelernt und im Gedächtnis abgelegt werden, in Anwendungssituationen vom Lerner jedoch nicht abgerufen und flexibel zur Lösung von Problemen eingesetzt werden können, d.h. die Personen verfügen über relevantes Wissen, setzen es aber nicht spontan ein. In diesem Sinne ist träges Wissen vergleichbar mit dem Begriff des Produktionsdefizits, wie er in der lernstrategisch orientierten Trainingsforschung beschrieben wurde (Brown \& DeLoache, 1978).

Die Verhinderung trägen Wissens ist ein zentrales Anliegen von Anchored Instruction (Bransfordet. et al., 1990; CTGV, 1990). Ausgangspunkt für die Entwicklung neuer Lernumgebungen der Vanderbilt-Gruppe waren Studien zur dynamischen Lernerfassung, die unter anderem zeigten, dass Schüler ihr Wissen und Kontextinformation bei der Lösung mathematischer Textaufgaben kaum nutzen (Bransford, Delclos, Vye, Burns \& Hasselbring, 1987; Bransfordet. et al. 1987; 1996). Auch andere Studien belegen, dass Rechenprozeduren oft blind, d.h. ohne kontextbezogene Überlegungen, eingesetzt werden (Charles \& Silver, 1988; Reusser, 1988).

Es werden verschiedene Gründe dafür geltend gemacht, dass die Anwendung und der Transfer von Wissen unter traditionellen Instruktionsbedingungen häufig zum Problem wird. So haben Lernende zum Beispiel oft wenig eigene Erfahrungen mit Problemtypen, die mit dem Wissen und den Fertigkeiten lösbar sind, die ihnen vermittelt werden (CTGV, 1997, 25). Wer erlebt es schon als echtes Problem, dass Winkel genau gemessen werden sollten? Wer kann wirklich nachvollziehen, dass Logarithmen eine wesentliche Erleichterung für die Lösung bestimmter mathematischer Probleme darstellen? Der Erwerb von Wissen und Fertigkeiten ist für viele Lernende eher ein Ziel in sich als ein Mittel zur Zielerreichung. Information, die bloss in Form von Fakten gespeichert ist, wird aber beim Problemlösen nicht unbedingt spontan aktiviert und genutzt (Simon, 
1980). Solches Wissen stellt kein brauchbares Werkzeug dar, sondern bleibt eben träge und eng mit der ursprünglichen Lernsituation verhaftet. Wird neues Wissen hingegen im Kontext bedeutsamer, problembasierter Lernaktivitäten erworben, kann eher erwartet werden, dass es später flexibel und instrumentell eingesetzt werden kann (CTGV, 1990, 1992b).

\section{Merkmale und Anwendungen von Anchored Instruction}

Der Begriff «Anchored Instruction» verweist auf das Grundprinzip, die angezielten Wissenskonzepte und Fertigkeiten in konkreten Problemlösekontexten zu verankern bzw. zu situieren. Dazu werden Makrokontexte in der Form von Videogeschichten geschaffen, die ein komplexes Problem exponieren, das bezüglich der Lösungswege eine gewisse Offenheit hat und dessen Bearbeitung die Definition verschiedener Teilprobleme erfordert (CTGV, 1992b). Die Lösung eines solchen Ankerproblems benötigt relativ viel Zeit (fachbezogene Lektionen über mehrere Tage oder Wochen). Der Makrokontext bietet auch Anlass zur vertieften, multiperspektivischen und fächerübergreifenden Exploration von Inhalten und Wissenskonzepten (CTGV, 1990). Anchored Instruction wird in engen Bezug gesetzt mit fallbasierten, problem- und projektorientierten Lernformen, in denen ebenfalls versucht wird, Wissen und Fertigkeiten mittels komplexer, authentischer Problemstellungen aufzubauen (CTGV, 1997, S. 47).

Die Vanderbilt-Gruppe hat in den letzten 12 Jahren vielseitige und komplexe Unterrichtsanker für verschiedene Fächer und Alterstufen geschaffen, die sich als multimedial unterstützte Lernumgebungen und -materialien präsentieren (CTGV, 1996a). Ihr Einsatz im schulischen Unterricht wurde erprobt, mit Interventionsstudien begleitet und evaluiert (CTGV, 1997; Sharp et al., 1995). In den folgenden Abschnitten werden Anwendungsfelder und Beispiele von Lernumgebungen näher beschrieben, damit sich der Leser eine konkretere Vorstellung davon machen kann. Danach werden Gestaltungsprinzipien, die für Anchored Instruction Lernumgebungen typisch sind erläutert und im Hinblick auf die Vor- und Nachteile des Einbezugs von Lerntechnologien diskutiert.

\section{Anwendungsfelder}

Die bekanntesten Unterrichtsanker wurden unter dem Titel «The Adventures of Jasper Woodbury” (kurz: Jasper Serie) für den Mathematikunterricht an mittleren und oberen Schulstufen entwickelt (CTGV, 1992a, 1997). Die Serie «Scientists in Action» bietet Anker für den naturwissenschaftlichen Unterricht (Goldman, Petrosino, Sherwood, Garrison, Hickey, Bransford, \& Pellegrino, 1996). Eine weitere Serie von Ankern, die "Little Planet Literacy Series», unterstützt den Aufbau von Lese- und Schreibfertigkeiten in den untersten Schulstufen (z.B. CTGV, 1996b). Neuere Entwicklungen betreffen Anker und technologisch ge- 
stützte Lern- und Reflexionsinstrumente für die Weiterbildung von Lehrpersonen oder Schulleitungsmitgliedern.

\section{Die Jasper Serie}

Zur Jasper Serie gehören eine beachtliche Anzahl von Ankerproblemen und technologisch gestützten Unterrichtsmaterialien und -instrumenten (CTGV, 1997, S. 1-17), die nachfolgend unter verschiedenen Gesichtspunkten vorgestellt werden.

\section{Präsentation und Bearbeitung von Jasper-Episoden}

Die Jasper Serie besteht insgesamt aus 12 kurzen Video-Episoden, in denen die Schüler jeweils am Schluss mit der Herausforderung konfrontiert werden, den Protagonisten der Geschichte zu helfen, ein komplexes Problem zu lösen. In der ersten Episode besichtigt und testet Jasper zum Beispiel ein gebrauchtes Motorboot, das sich ein gutes Stück flussaufwärts von seinem Wohnort entfernt befindet. Er will es kaufen und selber herrichten. Da die Bordbeleuchtung nicht funktioniert und es schon spät am Nachmittag ist, muss er sich überlegen, ob er es bis Sonnenuntergang schafft, das Boot nach Hause zu fahren.

Die Schüler werden dazu herausgefordert, das relativ komplexe Problem unter Berücksichtigung der im Film gegebenen Bedingungen und Daten (z.B. Dis$\operatorname{tanz}$, Zeit, Geschwindigkeit, etc.) zu lösen. Dies wird einerseits in Klassendiskussionen angeregt, andererseits erarbeiten sie gemeinsam in Gruppen mögliche Lösungen. Dazu werden verschiedene technologische Hilfsmittel benutzt: Die Videos werden auf Bildplatten oder CD-ROM präsentiert und mit einer Software können die Schüler jederzeit auf beliebige Video-Sequenzen zurückgreifen, wenn sie das komplexe Problem explorieren, zerlegen und die zur Lösung relevanten Angaben herausfiltern wollen.

\section{Aufgabentypen}

Mit Jasper-Problemen wird ein anwendungs- und verständnisorientierter Zugang zu Konzepten und Fertigkeiten in verschiedenen Mathematikbereichen angestrebt. Die Förderung mathematischen Denkens und der allgemeinen Problemlösefähigkeit steht im Vordergrund (CTGV, 1992a, 66). Schüler sollen lernen, (a) Teilprobleme zu definieren und zu mathematisieren, (b) lösungsrelevante von irrelevanten Hinweisen und Daten zu unterscheiden, (c) Daten, Operationen und Ergebnisse miteinander in Beziehung zu setzen, (d) zentrale mathematische Konzepte besser $\mathrm{zu}$ verstehen, und (e) Lösungen in einem kollaborativ-kommunikativen Kontext zu entwickeln. Diese Zielsetzungen spiegeln auch wider, dass bei der Entwicklung der Jasper Serie besonders auf die Umsetzung der Standards des «National Council of Teachers of Mathematics» geachtet wurde (NTCM, 1989). 
Jasper-Probleme sind nicht direkt auf die Festigung und Automatisierung von Prozeduren durch wiederholte Uebung ausgelegt. Die Serie will auch nicht das gesamte Mathematik-Curriculum ersetzen (CTGV, 1993a). Ein Jasper-Problem kann aber für Lehrpersonen und Schüler zum motivierenden Anlass werden, mathematische Prozeduren in diesem Kontext gezielt und vertieft zu instruieren bzw. zu üben. Es wird auch von «just-in-time instruction» gesprochen (CTGV, 1997, 3), weil Konzepte und Fertigkeiten, die im Hinblick aufs Curriculum zentral sind, im Unterricht in zeitlich und motivational günstigen Momenten aufgegriffen werden können.

Zur Unterstützung des Lerntransfers wurden die 12 Jasper-Episoden so konzipiert, dass jeweils drei mit unterschiedlicher Problemstellung und Komplexität im gleichen mathematischen Bereich angesiedelt sind, so dass die Bearbeitung mehrerer Probleme eine Vertiefung und Erweiterung von Konzepten und Fertigkeiten ermöglicht. Die ersten Jasper-Probleme (sogenannte «trip planning problems») können ca. ab der vierten Klasse bearbeitet werden. Sie zielen auf arithmetische Grundoperationen, Dezimal- und Prozentrechnung, Masskonzepte, Massumwandlungen u.a.m. Ein zweites Episoden-Tripel besteht aus Businessplan-Problemen, mit denen statistische Grundbegriffe und Vorgehensweisen eingeführt werden können. Die beiden letzten Tripel sind auf die Geometrie und die (Prä-)Algebra ausgerichtet.

Nach der Lösung der Ankerprobleme werden die Vertiefung, die Generalisierung und der Transfer durch das Bearbeiten analoger Probleme unterstützt, in denen gewisse Lösungsparameter verändert wurden. Erweiterte Aufgabenstellungen, die sich vom Ankerproblem deutlicher unterscheiden, dienen der zusätzlichen Flexibilisierung des Wissens (CTGV, 1997, 8). Als Erweiterung werden auch Klassenprojekte angeregt, die auf die Schüler und die Ressourcen in ihrem Umfeld zugeschnitten sind (Barron et al., 1995).

\section{Zusätzliche technologisch unterstützte Lernaktivitäten}

Für verschiedene Jasper-Episoden wurden sogenannte «SMART Challenges» und «SMART Tools» (SMART = Special Multimedia Arenas for Refined Thinking) entwickelt (Bransford et al., 1996; CTGV, 1997). SMART Challenges nutzen Kommunikationstechnologien (z.B. Videokonferenz, Internet) und interaktive CD-ROM, damit Schüler aus verschiedenen Regionen und Klassen ihre Jasper-Lösungen austauschen und vergleichen können. Die gesammelten Daten bieten auch Anlass für neue Aufgaben oder Hinweise, aus denen die Schüler allgemeinere Schlüsse ableiten können. Diese Zusatzaktivitäten sind an Jasper-Episoden verankert, führen aber darüber hinaus, indem sie die Diskussion und das Verständnis wichtiger mathematischer Konzepte unterstützen. SMART Tools sind mit dem Ankerkontext verbundene Mikrowelten, die softwarebasierte Planungs- und Visualisierungshilfen sowie Simulationen umfassen, mit denen die Schüler eigene Aufgaben und Arbeitswerkzeuge gestalten und erkunden können. Sie sollen Einsicht in bestimmte Zusammenhänge oder Gesetzmässigkeiten 
vermitteln und die allgemeine Planungs- und Reflexionsfähigkeit fördern.

Die SMART Erweiterungen wurden für die Schüler, aber auch für die Lehrpersonen geschaffen, damit sie anspruchsvollere Konzepte oder Prozeduren (z.B. Massstabtreue, Stichprobe, Funktionen, etc.) besser veranschaulichen, verstehen und sich darüber austauschen können. Sie sollen mathematisches Denken über eigene Modellbildung und über videobasierte, eingebettete Kurzinformationen und -instruktionen («embedded teaching») anregen (Bransford et al., 1996; CTGV, 1997).

\section{Ergebnisse aus Jasper-Untersuchungen}

Hier sollen nur kurz einige Ergebnisse aus Interventionsstudien mit der Jasper Serie erwähnt werden. Ausführlichere Darstellungen zur Entwicklung und den Ergebnissen der Jasper-Forschung finden sich in Publikationen der VanderbiltGruppe (Bransford et al., 1996; CTGV, 1997). In einer grossen Interventionsstudie zeigte sich erwartungsgemäss, dass "Jasper-Schüler» im Vergleich zu konventionell unterrichteten Schülern im Lösen von angewandten Textaufgaben und beim Planen von Lösungen überlegen waren. Im Erwerb mathematischer Konzepte und Fertigkeiten, die mit den Jasper-Problemen nicht vertieft geübt worden waren, erzielten sie während der Interventionszeit Fortschritte, die mit denjenigen der Kontrollgruppen vergleichbar waren. "Jasper-Schüler» zeigten nach der Intervention auch eine positivere Einstellung gegenüber der Mathematik. Die Erfahrungen und Einschätzungen der Lehrpersonen mit den Unterrichtsankern waren mit einer Ausnahme durchweg positiv.

\section{Die Little Planet Serie}

Die «Little Planet Literacy Series» besteht aus Trickfilm-Videos, die von Problemen fiktiver Tierfiguren auf einem kleinen Planeten erzählen. Mit den Ankergeschichten wird der Lese- und Schreibunterricht für Schulanfänger in semantisch komplexen, zielorientierten Lernaktivitäten situiert (CTGV, 1996b).

\section{Beispiel einer Ankergeschichte}

Die Geschichte «The Little Planet and the Magic Hats» thematisiert beispielsweise den Wert kritischen Beobachtens und Überprüfens von Sachverhalten, indem ein Scharlatan den Tieren für teures Geld Zauberhüte verkauft, die nichts taugen. Zum Glück gelingt es einem schlauen Tier, seinen Freunden mit objektiven Tests zu beweisen, dass sie einem Betrug zum Opfer gefallen sind, und das Geld kann zurückgefordert werden. Am Schluss der Geschichte werden die Kinder dazu herausgefordert, die relativ komplexe Geschichte selbst nachzuerzählen und aufzuschreiben, damit andere Tiere vor dem Betrüger gewarnt sind.

\section{Multimediale und konventionelle Lernaktivitäten}

Nach der Präsentation der Problemstellung besteht die erste Aktivität im Ordnen von Bildern aus der Geschichte in der richtigen Reihenfolge, was mit und 
ohne Computer erfolgen kann. Die Kinder können danach mit Unterstützung der Lehrperson und mit softwarebasierten Aktivitäten in Kleingruppen ein eigenes multimediales Buch auf dem Computer verfassen (CTGV, 1997, S. 145147). Auf jeder Buchseite steht oben ein Bild für einen Abschnitt der Geschichte. Per Mausklick kann im Bildfenster auch der betreffende Filmausschnitt abgespielt werden. Pro Buchseite machen die Kinder dann abwechselnd eigene Sätze zum Geschehen, die sie mit dem Computermikrophon aufnehmen. Ihre Kommentare können sie jederzeit abhören und verändern. Die Lehrperson und die Software unterstützen die Kinder schliesslich dabei, die mündlichen Kommentare zu verschriftlichen, indem einzelne Wörter und Sätze direkt über die Tastatur oder mit Hilfe des eingebauten, sprechfähigen Wörterbuchs eingegeben werden. $\mathrm{Zu}$ jeder Seite kann auch eine stimmige Melodie gewählt werden. Die multimedialen Bücher umfassen also stehende und animierte Bilder, gesprochenen und geschriebenen Text sowie musikalische Begleitung. Am Schluss werden die Bücher in einer elektronischen Klassenbibliothek publiziert, damit sie dort von allen Kindern angeschaut, gehört, gelesen und verglichen werden können.

Nebst softwarebasierten Aktivitäten gibt es auch viele Materialien, um Leseund Schreibaktivitäten zur Ankergeschichte mit Papier und Bleistift anzuregen. Das Lesen und Verfassen konventioneller Texte wird als sehr wichtig erachtet, weshalb der Transfer auf konventionelle Printmedien gefördert wird. So gibt es zum Beispiel von jeder Videogeschichte auch ein gedrucktes Textheft. Die multimedialen Bücher werden ebenfalls als Papierversion mit Bild und Text ausgedruckt, damit sie auch so gelesen, mitgenommen und mit anderen Kindern oder Erwachsenen geteilt werden können.

Die Präsentation und Bedienung der Software ist speziell auf jüngere Kinder abgestimmt und auf die Ankergeschichte bezogen. Dank multimedialen Erklärungen und Bedienungshilfen, die teilweise von Tieren aus der Geschichte geliefert werden, werden die vielen Programm-Optionen meist rasch gemeistert. Für Lehrpersonen gibt es eine spezielle Benutzerebene, um den Software-Zugang von Gruppen oder Individuen zu regeln, sowie ein Programm, das die von den Kindern gewählten Aktivitäten und deren Stand aufzeichnet.

\section{Analoge und erweiterte Aufgaben}

Die Lernumgebung enthält auch analoge Geschichten (z.B. der Betrug im Märchen «Des Kaisers neue Kleider»), die angeschaut, gelesen und nacherzählt werden können. Es gibt auch Verse über die Tiere auf dem kleinen Planeten, die zum Einüben wichtiger Grundwörter im Englischen eingesetzt werden. In allen softwaremässig aufbereiteten Texten können die Kinder Dekodier- oder Mitlesehilfen anfordern. So können beispielsweise Wörter markiert werden, die dann ausgesprochen und gleichzeitig auditiv und visuell in Silben getrennt werden. Zusätzliche softwarebasierte oder konventionelle Aktivitäten bestehen aus dem Fortsetzen von Geschichtenanfängen und dem freien Gestalten eigener Geschichten und Bilder. 
In einer Pilotstudie, in der bei Schulanfängern ein integriertes Curriculum für Lesen und Schreiben, Mathematik und naturwissenschaftliche Themen erprobt wurde (CTGV, 1996c), wurde eine Software-Erweiterung studiert, die den Übergang von narrativem zu expositorischem Text unterstützt und das Hauptthema einer Ankergeschichte nutzt, um eine Brücke zu naturwissenschaftlichen Inhalten zu schlagen (Weise, 2001; Weise und Scharnhorst, 2001a).

\section{Ergebnisse zu Little Planet-Studien}

Bisherige Ergebnisse mit der Little Planet Serie beziehen sich auf einige Schulklassen, in denen das oben erwähnte integrierte Curriculum ausprobiert wurde (z.B. CTGV, 1996c). Die Ergebnisse zu den Lese- und Schreibleistungen der Kinder werden hier nur zusammenfassend erwähnt. Im Gegensatz zu Kontrollgruppen haben Kinder, die mit der Little Planet Lernumgebung lernten, deutlich bessere Leistungen beim freien Schreiben kurzer Texte erzielt, die mit einem Bild und einem Eingangssatz angeregt wurden. Sie schrieben mehr und strukturierten ihre Texte besser. In einem standardisierten Lesetest schnitten sie gut ab, unterschieden sich jedoch nicht signifikant von den Kontrollgruppen.

\section{Designprinzipien}

Bevor der Einbezug technologischer Unterstützungsformen im Unterricht näher diskutiert wird, sollen kurz die Designprinzipien vorgestellt werden, die in Bezug auf die Gestaltung der Jasper-Serie formuliert wurden (CTGV, 1992a, 1997; Mandl, Gruber \& Renkl, 1995). Sie gelten mit wenigen Einschränkungen auch für Anwendungen von Anchored Instruction, die nicht auf Mathematik bezogen sind. Nach der vorangehenden Beschreibung der Lernumgebungen erscheinen sie relativ evident.

(1) Videobasiertes Präsentationsformat

(2) Narratives Präsentationsformat

(3) Generatives Lernformat (eigene Problemdefinitionen sind nötig)

(4) Eingebettete Daten (Lösungsbedingungen und-daten sind im Video eingebettet)

(5) Problemkomplexität

(6) Episoden-Tripel

(7) Fächerübergreifende Elemente

Einige der Vorteile, die mit diesen Designprinzipien und dem Einsatz entsprechender Lerntechnologien verbunden sind, sollen kurz aufgeführt werden. Sie beziehen sich auf (a) das Präsentationsformat der Probleme, (b) deren individuelle und soziale Repräsentation und auf (c) die Lern- und Lehraktivitäten, die damit unterstützt werden:

a. Das videobasierte narrative Format ermöglicht die dynamische und realitätsnahe Präsentation von komplexen, motivierenden Problemen in kurzer Zeit, 
weil die Informationen multimedial und eher simultan (als sequentiell) angeboten werden können (Bransford et al., 1990).

b. In Bezug auf die individuelle Problemrepräsentation wird der Aufbau mentaler Situationsmodelle und der Zugang für sprachlich schwächere Schüler erleichtert (Sharp et al., 1995). Die Videos zeigen auch positive Rollenmodelle für kollaboratives Problemlösen.

In Bezug auf die soziale Repräsentation der Probleme schaffen Videoanker einen gemeinsamen Kontext, den alle Bezugspersonen leicht teilen können und der allen gleiche Vorinformationen liefert. Informations- und Kommunikationstechnologien ermöglichen die weitere Vernetzung von Lernenden und Lehrenden (CTGV, 1997).

c. Für die Lernenden erleichtert der Zugang zu beliebigen Videosequenzen die wiederholte und aktive Problemexploration. Eingebaute Hilfe- und Feedbakkfunktionen ermöglichen aktives, teilweise sogar interaktives Lernen, das nicht unbedingt von der Lehrperson abhängt. Erworbenes Wissen kann in Transferaufgaben unter multiplen Perspektiven eingesetzt werden. Die softwaregestützten Aktivitäten ermöglichen das Herstellen ansprechender und kommunzierbarer Produkte.

Für die Lehrpersonen erweitern flexibel adaptierbare Technologien, die Möglichkeiten individuelle Lernhilfen zu geben und gruppenorientierte Projekte durchzuführen. Die Ankerprobleme sichern aber gleichzeitig einen kollektiven, klassenbezogenen Fokus. «Embedded teaching»-Funktionen helfen, komplexe Inhalte in geeigneten Momenten zu aktualisieren und zu vermitteln (CTGV, 1997). Transferorientierter Unterricht wird mit dem Angebot analoger und erweiterter Aufgaben erleichtert. Das Einüben basaler Fertigkeiten kann im Kontext komplexer, bedeutungsvoller Problemstellungen erfolgen. Auf dem Computer bleiben Lernprodukte und Prozessdaten von Individuen oder Gruppen erhalten, die auch nach dem Unterricht eingesehen werden können.

\section{Kombinationen sozialer und technologischer} Unterstützungsformen beim Lernen

In Anchored Instruction Implementationen sind vielfältige Formen der Lernanregung und -unterstützung vorgesehen. Mit den Designprinzipien wird versucht, lern- und transferfreundliche Makrokontexte zu gestalten. Andererseits wird das Lernen auf der individuellen Ebene mit sozialen (Peer-Kollaboration, Lehrerunterstützung) und maschinellen Stützfunktionen (interaktive Lerntechnologien) gefördert. An dieser Stelle muss noch angefügt werden, dass die Vanderbilt-Gruppe in ihren Projekten mit den beteiligten Lehrkräften meist intensiv zusammenarbeitet und deren professionelle Weiterbildung stark unterstützt (CTGV, 1997, S. 109-119). Dies hat sich auch als notwendig erwiesen, weil der fruchtbare Einsatz der Lernumgebungen zu Beginn kaum ohne viel persönlichen und technischen Support möglich ist. 


\section{Eine Taxonomie von Stützfunktionen}

Im Rahmen einer Pilotstudie, in der die Little Planet Lernumgebung als Grundlage für ein integriertes Curriculum genutzt wurde, schlägt Weise (2001) eine Taxonomie von Stützfunktionen («scaffolds») vor, die auch für andere Anchored Instruction-Umsetzungen charakteristisch erscheinen:

Als Mikro-Stützfunktionen werden direkte, problem- und lernerbezogene Hilfestellungen bezeichnet, während Makro-Stützfunktionen eher indirekte lernunterstützende Bedingungen auf der Ebene der gesamten Lernumgebung schaffen. Mikro- und Makro-Stützfunktionen können einzeln, nacheinander oder gleichzeitig wirksam sein.

Beispielsweise helfen Mikro-Stützfunktionen einem Lerner, eine Textstelle oder Teilaufgabe zu bewältigen. Denkbar sind person- oder softwarebasierte Lernhilfen, die direkt, individuell und intermittierend angeboten werden. Idealerweise liegen sie in der Zone der proximalen Entwicklung des Lerners ( $\mathrm{Vy}-$ gotsky, 1978). Für Beobachter sind sie in der Regel erkennbar.

Im Gegensatz dazu sind Makro-Stützfunktionen nicht primär auf die Bewältigung individueller Lernhindernisse ausgerichtet, sondern auf die Bereitstellung günstiger Rahmenbedingungen, in denen intentionales, konstruktives Lernen stattfinden kann. In einer optimal gestalteten Umgebung, die dem Entwicklungsstand der Lernenden angemessen ist, sind Makro-Stützfunktionen kontinuierlich über längere Zeiträume vorhanden und unterstützen den Zugang zu Lerninhalten und Lernprozessen. Aussenstehenden fallen sie nicht unbedingt als besondere Formen der Unterstützung auf.

Beide Kategorien können weiter differenziert werden (Weise, 2001): MikroStützfunktionen sind direkte Lernhilfen, die innerhalb oder ausserhalb des Aufgabenkontexts lokalisiert sein können, in dem ein Lerner gerade aktiv ist (intravs. extrakontextuell).

Eine intrakontextuelle Mikro-Stützfunktion ist zum Beispiel gegeben, wenn die Lehrperson Schüler mit geeigneten Fragen unterstützt, damit sie ein vorgängig gezeigtes Experiment korrekt interpretieren. Liest ein Lerner einen Bildschirmtext und kann dabei auf eine eingebaute Dekodierhilfe zugreifen, so handelt es sich ebenfalls um eine intrakontextuelle Hilfe.

Extrakontextuelle Mikro-Stützfunktionen sind aufgabendienliche Hinweise, die ausserhalb des direkten Lernfeldes liegen. Häufig ist damit ein Wechsel von einem physikalischen zu einem digitalen Kontext oder umgekehrt verbunden. Ein Beispiel dafür sind verbale Lehrerhinweise, die einem Schüler helfen, eine Aufgabe auf dem Computer zu lösen. Ein anderes Beispiel ist ein Schüler, der einen Text von Hand schreibt und die Schreibweise einiger Wörter mit Hilfe eines elektronischen oder konventionellen Lexikons prüft.

Wie oben erwähnt, stellen Makro-Stützfunktionen grundsätzlich lernförderliche Bedingungen bereit. Daneben werden ihnen zwei besondere Wirkweisen zugeschrieben, die in gegenseitigem Bezug stehen: Vertraute Makrokontexte un- 
terstützen einerseits den Zugang zu neuen, andersartigen Aufgaben ( inter-task scaffolds»), andererseits können vertraute Aufgaben aus einem Makrokontext herausgelöst werden, um den Übergang zu neuen Lernumgebungen zu erleichern ("inter-environmental scaffolds»).

In der Studie von Weise (2001) diente eine den Kindern vertraute Videogeschichte und die damit verbundene Lernumgebung als «inter-task scaffold», um den Wechsel von narrativen zu expositorischen Texten zu erleichtern. Sachtexte sind mit neuen Aufgaben verbunden, die Schülern in der Grundschule oft Schwierigkeiten bereiten. Informationen müssen nun im Hinblick auf bestimmte Ziele oder Fragen selegiert, kombiniert und als Bericht, nicht als Erzählung, gelesen bzw. verfasst werden. Sobald die Schüler mit diesen neuen Aufgaben in der alten Lernumgebung vertraut gemacht worden waren, wurde dies als «inter-environmental scaffold» genutzt, um sie mit erweiterten Aufgabenstellungen in einer neuen Lernumgebung zu konfrontieren. Die neue, softwaregestützte Lernumgebung "NoteTaker» bezog sich nur noch auf Sachtexte und bot eine Reihe von Mikro-Stützfunktionen, welche den Schülern helfen, ihre Fertigkeiten im Umgang mit expositorischen Texten zu entwickeln und zu vertiefen.

\section{Makrokontexte und Mikrowelten}

Beim Design von Anchored Instruction Lernumgebungen wird viel in die Realisierung von Makro- und Mikrostützfunktionen investiert. Dabei wird eine Kombination von "Macrocontexts Plus Microworlds» favorisiert, weil dies für bewusstes und nachhaltiges Lernen über längere Zeiträume als günstig erachtet wird (Crews et al., in press): Einerseits fördern Makrokontexte und Mikrowelten als multiple Perspektiven die Generalisierung und den Transfer, andererseits setzt sich die Beziehung zwischen Makrokontexten und Mikrowelten fort in der Beziehung zwischen Mikrowelten und der Konstruktion individueller kognitiver Werkzeuge.

Diese Designstrategie unterschiedet sich von einem «basics first» Ansatz, der auf einer «bottom-up» Strategie und der Annahme beruht, dass zuerst basale Fertigkeiten in reduzierten Lernkontexten eingeübt werden müssen, bevor diese kombiniert und in komplexere Anwendungskontexte umgesetzt werden können (Crews et al., in press). Auch andere Autoren kritisierten das Aufbrechen komplexer Aufgaben wie Lesen oder Rechnen in einfache, isolierte Teilkomponenten und das Training mit dekontextualisierten Übungsformen (Brown et al., 1991): Es zeigt sich, dass das Zusammensetzen der einzelnen Elemente (vertikaler Transfer) oder deren Nutzung in verschiedenen Anwendungssituationen (lateraler Transfer) im Sinne Gagnés (1965) meist nicht gelingen, weil sie den Lernenden überlassen werden.

In Anchored Instruction Lernumgebungen (und anderen konstruktivistisch gestalteten Lernkontexten) werden die Lernenden von Anfang an mit ganzheitlichen, anspruchsvollen Aufgaben konfrontiert. Damit sie bewältigt werden kön- 
nen, müssen Makro- und Mikro-Stützfunktionen bewusst und gezielt eingeplant werden. Im Sinne des Lernens in Zonen der proximalen Entwicklung (Vygotsky, 1978) werden also nicht die Aufgabenstellungen vereinfacht, sondern vielmehr kognitive Stützfunktionen (Wood, Bruner \& Ress, 1976 ) für die Lernenden konzipiert. Dazu gehören sozial-interaktive Lehr- und Lernformen, die auf die Zusammenarbeit zwischen Experten und Novizen oder Peers bauen. Bei Anchored Instruction wird die Bewältigung komplexer Aufgaben mit sophistizierten technologischen Anwendungen zusätzlich unterstützt. Mit sozialen und technologischen Mikro-Stützfunktionen wird das Training von Grundfertigkeiten zum Teil direkt in die Bearbeitung komplexer Probleme integriert. Die Verbindung von Makrokontexten mit Mikrowelten vereint top-down- und bottomup-Strategien, indem den Lernenden sowohl Zugänge über komplexe Aufgaben im Makrokontext als auch über individuelle Lernaktivitäten in computergestützten Mikrowelten eröffnet werden (Crews et al., in press).

\section{Kritische Fragen zu Anchored Instruction Lernumgebungen}

Es gibt verschiedene kritische Fragen zu Anchored Instruction Lernumgebungen. Im Folgenden sollen einige davon unter Einbezug von Diskussionen in der Fachliteratur, Untersuchungsergebnissen sowie eigenen Erfahrungen und Überlegungen diskutiert werden.

\section{Sind die Aufgaben situiert, realitätsbezogen und authentisch?}

Der Anspruch, dass mit videobasierten, narrativen Ankern situierte, realitätsbezogene und authentische Probleme gestellt werden, hat Anlass zu Kritik gegeben (Tripp, 1996). Zuweilen wird auch die generelle Position vertreten, dass der Einsatz von Lerntechnologien die Realität nicht näher, sondern zum Verschwinden bringt (von Hentig, 1984). Dahinter steht im Grunde die Auffassung, dass situiertes Lernen auf reales Problemlösen in realen Alltagssituationen beschränkt ist. Die Vertreter situierten Lernens gehen jedoch nicht davon aus, dass verbal-propositional basiertes Problemlösen in der Schule im Gegensatz zu praktisch-handelndem Problemlösen im Alltag grundsätzlich nicht situiert sei. Vielmehr geht es darum, dass die Merkmale des «learning by doing» unter Anleitung von Experten in informellen Lernsituationen im Kontext schulischen Lernens vermehrt realisiert werden (Brown et al., 1989).

Dennoch muss die Frage gestellt werden, was in welcher Form für wen authentisch ist. Die Vanderbilt-Gruppe nennt drei Kriterien für Authentizität (CTGV, 1990, S. 7): (a) faktischer Realitätsbezug in den Ankergeschichten, (b) Authentizität der Probleme für die Lernenden, (c) Authentizität bezüglich der angezielten Lerninhalte. 
Zugegebenermassen erfüllen nicht alle Unterrichtsanker alle Kriterien. Bei der Jasper Serie ist der faktische Realitätsbezug relativ hoch, d.h. die in den Geschichten dargestellten Personen, Objekte und Situationen wirken realistisch, sind aber auf amerikanische Verhältnisse bezogen, was ihre Authentizität für Lernende in anderen sozio-kulturellen Kontexten einschränkt. Die Jasper-Probleme können auch als authentisch für die Lernenden angesehen werden, weil Hauptund Teilprobleme aufeinander bezogen sind und sich direkt aus den Geschichten heraus ergeben. Verzwickte Abenteuer entsprechen auch den altersmässigen Interessen der Lernenden. Im Gegensatz dazu sind eingekleidete Textaufgaben oft wenig authentisch (z.B. Zug A fährt um die Zeit B von X nach Y...usw. ). Die Authentizität von Jasper-Problemen in Bezug auf mathematische Problemstellungen und Erfahrungen mag zum Teil fragwürdig sein, weil in erster Linie allgemeine Problemlöse-Situationen gestellt werden und erst durch deren Bearbeitung mathematische Bezüge hergestellt werden (CTGV, 1990; 1993a). Deshalb wurden später auch die SMART Erweiterungen entwickelt.

Die Little Planet Serie hat im Grunde keinen faktischen Realitätsbezug, weil es sich um fiktive Figuren und Trickfilmgeschichten handelt. Andererseits entsprechen solche Geschichten der Erlebniswelt junger Kindern, und die behandelten Themen (z.B. Betrug) verweisen, ähnlich wie Fabeln, auf die Realität. Die Kinder identifizieren sich meist spontan mit dem Auftrag, die Geschichte nachzuerzählen, der folglich zu Beginn als authentisch empfunden wird. Das Hintergrundziel, nämlich die Lese- und Schreibfähigkeiten zu entwickeln, wird dabei an den Zweck der Sicherung und des Austauschs von Information gebunden. Da die Umsetzung des Auftrags nur in einem längeren Zeitraum realisiert werden kann, muss man sich aber fragen, ob die Authentizität der Aufgabe und der Lernaktivitäten tatsächlich erhalten bleibt.

\section{Ist der Einsatz technologischer Mittel notwendig?}

Ein Punkt, der oft angesprochen wird, ist die Frage, inwieweit situierte Lernumgebungen im schulischen Kontext auf den Einsatz technologischer Mittel zurükkgreifen müssen oder sollen. Die Umsetzung der Designprinzipien von Anchored Instruction erfordern den Einsatz multimedialer Lerntechnologien. Videobasiertes, narratives, generatives, komplexes Problemformat und eingebettete Daten können mit Printmedien kaum realisiert werden (CTGV, 1993a; Mandl, Gruber \& Renkl,1995). Einerseits entsprechen videobasierte Lernumgebungen dem europäischem Empfinden vielleicht weniger und lösen mehr Bedenken aus als dies in den USA der Fall ist, andererseits mögen die Widerstände in unserem Sprachraum zum Teil auch auf eine gewissen Technikphobie im schulischen Bereich zurückzuführen sein (Kohler, 1998).

Es muss aber berücksichtigt werden, dass multimediale technologisch unterstützte Lernumgebungen, die den Anforderungen situierten Lernens entsprechen, einen riesigen Erstellungsaufwand und - was ebenso wichtig ist - eine 
sorgfältige didaktische Einbettung erfordern (Mandl, Gruber \& Renkl,1995). Dass sich die Vanderbilt-Gruppe diesbezüglich viele Überlegungen macht, zeigen die geschilderten Bemühungen zur Kombination sozialer und technologischer Unterstützungsformen, aber auch die vielfältigen Transfer-Aufgaben und projekte, die über die Unterrichtsanker hinausführen.

Eine wichtige Frage, die von den Vertretern des Anchored Instruction Ansatzes bisher kaum direkt thematisiert und erforscht wurde, betrifft die Abhängigkeit des Lernerfolgs von Lernermerkmalen (ATI-Effekte) in komplexen, technologisch gestützten Lernumgebungen. Mandl, Gruber \& Renkl (1995) weisen darauf hin, dass einige Studien nahelegen, dass sich Lernerfolge in solchen Umgebungen eher einstellen, wenn Lerner bereits über ein höheres Leistungs- oder Fertigkeitsniveau und eine konstruktivistische Lernauffassung verfügen.

Ergebnisse aus Jasper-Untersuchungen liefern indirekt einige Argumente gegen die Annahme von ATI-Effekten bei Anchored Instruction. Der Einsatz von Jasper-Problemen in Verbindung mit SMART Erweiterungen erzeugte im Vergleich zur blossen Bearbeitung von Jasper-Probleme einen zusätzlichen Gewinn in den leistungs- und motivationsbezogenen Gruppenmittelwerten (CTGV, 1997). Dabei waren in der untersuchten Population auch 20-25\% Schüler, die in der Mathematik als besonders leistungsschwach galten. Eine weitere Intervention mit Jasper + SMART zeigte, dass beachtliche Leistungssteigerungen zwischen Prä- und Posttest bei den Schülern im unteren, mittleren und oberen Leistungsbereich zu verzeichnen waren. Die Unterschiede zwischen den Leistungsgruppen blieben auch nach der Intervention erhalten, doch zeigten sich keine Interaktionen zwischen anfänglichem Leistungsniveau und Leistungszuwachs, so dass man davon ausgehen kann, dass alle Schüler vom Programm gleichermassen profitierten. Auch Erfahrungen und qualitative Berichte von Lehrpersonen weisen darauf hin, dass Lernumgebungen wie Jasper und Little Planet für schwächere Schüler besonders geeignet sind.

\section{Problem- oder projektbasierte Lernformen?}

Auch wenn Anchored Instruction Umgebungen die Anforderungen situierten Lernens erfüllen und didaktisch überlegte und sinnvolle Anwendungen der Lerntechnologie darstellen, kann man sich fragen, ob mit handlungsorientierten Lernprojekten mit weniger Aufwand nicht ähnliche Ziele verfolgt und ebenso fruchtbare Ergebnisse erzielt werden könnten. Die Vanderbilt-Gruppe betrachtet ihre Ankervideos und die technologisch gestützten Lernumgebungen als Einstieg ins eigenaktive Lernen mit Bezug auf bestimmte Lernziele und Fächer, dem idealerweise eigene Lernprojekte folgen (z.B. CTGV, 1993a). Davon abgesehen, werden im Einsatz von Unterrichtsankern gegenüber Lernprojekten verschiedene Vorteile gesehen: (1) Für Lehrpersonen ist der Unterricht mit Ankern meist einfacher zu organisieren als mit Projekten. (2) Anker bieten offene Problemräume, sichern aber gleichzeitig, dass die Schüler sich mit intendierten Lerninhalten befassen. Jasper-Episoden wurden beispielsweise so konzipiert, dass darin 
mathematische Konzepte und Fertigkeiten aufgegriffen werden, die im altersgemässen Curriculum wichtig sind. (3) Anker schaffen einen gemeinsamen Hintergrund, der mit den Videos auch gegen aussen leicht vermittelt werden kann. (4) Anker bieten eine Basis für sinnvolle formative Evaluationen.

Ausserdem zeigen Untersuchungsergebnisse, dass problembasiertes Lernen mit Jasper-Episoden und SMART Erweiterungen den Übergang zu projektbasiertem Lernen erleichert und die Leistungen von Schülern in Lernprojekten erhöht (CTGV, 1997). Das ist zu einem grossen Teil auch darauf zurückzuführen, dass bei der Lösung von Jasper-Problemen in Kleingruppen, die Fähigkeit der Schüler zur produktiven, zielorientierten Teamarbeit aufgebaut werden kann. Dies spiegelt auch wider, dass Vertreter des Situiertheitsansatzes unter Lernen die erhöhte Fähigkeit verstehen, mit Objekten und andern Personen in Situationen zu interagieren. Transfer bedeutet dann, dass die Fähigkeit in einer bestimmten Situation an einer Aktivität zu partizipieren auf die Teilhabe an andern Aktivitäten in neuen Situationen übertragen werden kann (Greeno et al., 1993).

\section{Schlussfolgerungen}

Zweifellos könnten noch weitere Punkte in Bezug auf Anchored Instruction kritisch diskutiert werden. Abschliessend sollen nochmals diejenigen Aspekte hervorgehoben werden, die besonders wichtig erscheinen.

Anchored Instruction Lernumgebungen gehören im schulischen Bereich sicher zu den am weitesten entwickelten Umsetzungen situierten Lernens mit technologischer Unterstützung. Sie realisieren eine grosse Anzahl der gestellten Forderungen und ihr Einsatz hat in verschiedensten Kontexten zu überaus positiven Ergebnissen geführt. Die Arbeiten der Vanderbilt-Gruppe haben auch viel zur Diskussion und Entwicklung des Situiertheitsansatzes beigetragen.

Daneben gibt es aber auch eine Reihe begründeter Bedenken und einschränkender Bedingungen im Zusammenhang mit Anchored Instruction. Die Erstellung solcher Lernumgebungen erfordert einen enormen personellen und technischen Aufwand, der nur von grossen multidisziplinären Teams geleistet werden kann. Da die problembasierten Unterrichtsanker im sozio-kulturellen Umfeld der potentiellen Lerner situiert werden müssen, wird ihre Anwendung in anderen Kontexten gleichzeitig begrenzt. Auch die Implementation und Evaluation solcher Lernumgebungen im schulischen Umfeld stellt eine Anzahl nicht zu unterschätzender Probleme. In der Regel handelt es sich um komplexe, nur teilweise überschaubare "Designexperimente» in natürlichen Kontexten, die Forschende mit vielen methodischen Schwierigkeiten konfrontieren (Brown, 1992). Die dabei anfallenden Daten sind oft nur schwer zu interpretieren und zu generalisieren. 
Auch wenn bei der Gestaltung der Lernumgebungen viele Vorkehrungen getroffen werden, damit diese konstruktivistisches und situiertes Lernen begünstigen, ist damit natürlich noch nicht garantiert, dass Lehrende und Lernende sie auch auf eine Weise nutzen, die der Intention entspricht. Die Rollen von Lehrenden und Lernenden müssen geklärt und auch gelebt werden, sie werden nicht allein durch die Materialien induziert. Die Vanderbilt-Gruppe hat auch darauf hingewiesen, das ihre Lernumgebungen mit unterschiedlichen Unterrichtsmodellen und didaktischen Überlegungen genutzt werden können, die nicht alle in gleichem Masse den Forderungen konstruktivistischen Lernens entsprechen (CTGV, 1997, S. 56-62).

Es hat sich auch gezeigt, dass Lehrpersonen bei der Implementation unter Umständen relativ viel Unterstützung und gegenseitigen Austausch benötigen, was die Verantwortlichen der Implementation vor zusätzliche Herausforderungen stellt. Da es keine Langzeitstudien gibt, ist beispielsweise auch nicht klar, wie sich die Nutzung der technologisch gestützten Lernumgebungen an Schulen nach der forschungsmässig begleiteten Phase weiterentwickelt. Es muss aber auch positiv hervorgehoben werden, dass viele Implementationen absichtlich nicht unter besonders förderlichen Bedingungen gemacht wurden, sondern an öffentlichen Schulen mit ungünstigen infrastrukturellen Bedingungen und Schülerpopulationen, die aus sozio-kulturell benachteiligten Schichten stammen. Die Erfahrung, dass auch unter diesen schwierigen Bedingungen konstruktive (und konstruktivistische) Lernprozesse ausgelöst, beobachtet und dokumentiert werden konnten, spricht für diese Lernumgebungen. Ihr künftiger Einsatz und ihre Weiterentwicklung wird zeigen, ob sie sich in der Unterrichtspraxis bewähren und uns auch erlauben, auf der theoretischen Ebene neue Erkenntnisse über den Erwerb und Transfer von Wissen beim situierten Lernen zu gewinnen.

\section{Anmerkungen}

1 Der Begriff «Anchored Instruction» (= verankerte(r) Instruktion, Unterricht) wird meist nicht übersetzt, weil damit der direkte Bezug zu den Arbeiten des Learning Technology Center angesprochen wird.

2 Das LTC ist ein Forschungszentrum am Peabody College der Vanderbilt University. Es umfasst eine grössere Anzahl Mitarbeiter, die auf dem Hintergrund ihrer jeweiligen Fachdisziplinen und Spezialisierungen in interdisziplinären Teams technologisch unterstützte Lernumgebungen entwickeln und erforschen. Die Arbeiten des LTC erscheinen häufig als Gemeinschaftspublikationen, unter dem Namen «Cognition and Technology Group at Vanderbilt (CTGV).

3 Unter dem Begriff «authentisch» wird in erster Linie die Bedeutsamkeit und Attraktivität der Problemstellungen für die Lernenden verstanden. Das heisst nicht, dass es sich um Falldarstellungen oder Probleme handelt, die sich tatsächlich ereignet haben oder ereignen könnten. Das Problem der Authentizität wird später im Text detaillierter diskutiert. 


\section{Bibliographie}

Barron, B. J., Vye, N. J., Zech, L., Schwartz, D, Bransford, J. D., Goldman, S. R., Pellegrino, J., Morris, J., Garrison, S. \& Kantor, R. (1995). Creating contexts for community-based problem solving: The Jasper challenge series. In C. N. Hedley, P. Antonacci \& M. Rabinowitz (Eds.), Thinking and literacy: The mind at work (pp. 47-71). Hillsdale, NJ: Erlbaum.

Bransford, J. D., Delclos, V, Vye, N., Burns, S. \& Hasselbring, T. (1987). Approaches to dynamic assessment: Issues, data, and future directions. In C. Lidz (Ed.), Dynamic assessment: An interactional approach to evaluating learning potentials (pp. 479-495). New York: Guilford.

Bransford, J. D., Franks, J. J., Vye, N.J. \& Sherwood, R. D. (1989). New approaches to instruction: Because wisdom can't be told. In S. Vosniadou \& A. Ortony (Eds.), Similarity and analogical reasoning (pp. 470-497). New York: Cambridge University Press.

Bransford, J. D., Sherwood, R. D., Hasselbring, T. S., Kinzer, C. K. \& Williams, S. M. (1990). Anchored instruction: Why we need it and how technology can help. In D. Nix \& R. Spiro (Eds.), Cognition, education and multimedia: Exploring ideas in high technology (pp. 115-141). Hillsdale, NJ: Erlbaum.

Bransford, J. D., Zech, L. Schwartz, D., Barron, B., Vye, N. \& CTGV (1996). Fostering mathematical thinking in middle school students: Lessons from research. In R. J. Sternberg \& T. Ben-Zeev (Eds.), The nature of mathematical thinking (pp. 203-250). Mahwah, NJ: Erlbaum.

Brown, A. L, (1992). Design experiments: Theoretical and methodological challenges in creating complex interventions in classroom settings. The Journal of the Learning Sciences, 2, (2), 141-178.

Brown, A. L. \& Campione, J. C. (1994). Guided discovery in a community of learners. In K. M. Gilly (Ed.), Classroom lessons: Integrating cognitive theory and classroom practice (pp. 229-272). Cambridge, MA: MIT Press/Bradford Books.

Brown, A. L., Campione, J. C., Reeve, R. A., Ferrara, R. A. \& Palincsar, A. S. (1991). Interactive learning and individual understanding: The case of reading and mathematics. In L. T. Landsman (Ed.), Culture, schooling and psychological development (pp. 136-170). Hillsdale, NJ: Erlbaum.

Brown, J.S., Collins, A. \& Duguid, P. (1989). Situated cognition and the culture of learning. Educational Researcher, 18, (1), 32-42.

Brown, A. L. \& DeLoache, J. S. (1978). Skills, plans, and self-regulation. In R. S. Siegler (Ed.), Children's thinking: What develops? (pp. 3-36). Hillsdale, NJ: Erlbaum.

Charles, R. \& Silver, E. A. (Eds.) (1988). The teaching and assessing of mathematical problem solving. Hillsdale, NJ: Erlbaum \& National Council for Teachers of Mathematics.

Cognition and Technology Group at Vanderbilt (1990). Anchored instruction and its relationship to situated cognition. Educational Researcher, 19, (6), 2-10.

Cognition and Technology Group at Vanderbilt (1992a). The Jasper Experiment: An exploration of issues in learning and instructional design. Educational Technology Research and Development, 40, 65-80.

Cognition and Technology Group at Vanderbilt (1992b). The Jasper series as an example of anchored instruction: Theory, program description, and assessment data. Educational Psychologist, 27, 291-315.

Cognition and Technology Group at Vanderbilt (1993a). Anchored Instruction and situated cognition revisited. Educational Technology, 33, 52-70.

Cognition and Technology Group at Vanderbilt (1993b). Designing learning environments that support thinking: The Jasper series as a case study. In T. M. Duffy, J. Lowyck \& D. Jonassen (Eds.), Designing learning environments for constructive learning (pp. 1-36). New York: Springer. 
Cognition and Technology Group at Vanderbilt (1993c). Toward integrated curricula: Possibilities from anchored instruction. In M. Rabinowitz (Ed.), Cognitive science foundations of instruction (pp. 33-55). Hillsdale, NJ: Erlbaum.

Cognition and Technology Group at Vanderbilt (1994a). Multimedia environments for developing literacy in at-risk students. In B. Means (Ed.), Technology and education reform: The reality behind the promise (pp. 23-56). San Francisco: Jossey-Bass.

Cognition and Technology Group at Vanderbilt (1994b). From visual word problems to learning communities: Changing conceptions of cognitive research. In K. McGilly (Ed.), Classroom lessons: Integrating cognitive theory and classroom practice (pp. 157-200). Cambridge, MA: MIT Press.

Cognition and Technology Group at Vanderbilt (1996a). Looking at technology in context: A framework for understanding technology and education research. In D. C. Berliner \& R. C. Calfee (Eds.), The handbook of educational psychology (pp. 807-840). New York: MacMillan.

Cognition and Technology Group at Vanderbilt (1996b). A multimedia literacy series that celebrates authorship and books. Communications of the Association for Computing Machinery (ACM), 39, (8), 106-109.

Cognition and Technology Group at Vanderbilt (1996c). Year 1 Report to the James S. McDonnell Foundation. Unpublished report, Learning Technology Center, Vanderbilt University, Nashville.

Cognition and Technology Group at Vanderbilt (1997). The Jasper project. Lessons in curriculum, instruction, assessment, and professional development. Mahwah, NJ: Erlbaum.

Crews, T. R., Biswas, G., Goldman, S. \& Bransford, J. D. (in press). Macrocontexts plus microworlds: an anchored instruction approach to intelligent learning environments. Journal of $\mathrm{AI}$ in education.

Duffy, T. M. \& Jonassen, D. H. (Eds.) (1992). Constructivism and the technology of instruction. Hillsdale, NJ: Erlbaum.

Gagné (1965). The conditions of learning. New York: Holt, Rinehart \& Winston.

Gerstenmaier, J. \& Mandl, H. (1995). Wissenserwerb unter konstruktivistischer Perspektive. Zeitschrift für Pädagogik, 41, 6, 867-888.

Goldman, S. R., Petrosino, A., Sherwood, R. D., Garrison, S., Hickey, D., Bransford, J. D. \& Pellegrino, J. W. (1996). Anchoring science instruction in multimedia learning environments. In S. Vosniadou, E. De Corte, R. Glaser \& H. Mandl (Eds.), International perspectives on the design of technology-supported learning environments (pp. 257-284). Hillsdale, NJ: Erlbaum.

Greeno, J. G., Smith, D. R. \& Moore, J. L. (1993). Transfer of situated learning. In D. K. Detterman \& R. J. Sternberg (Eds.), Transfer on trial: Intelligence, cognition, and instruction (pp. 99-167). Norwood, NJ: Ablex.

Kohler, B. (1998). Problemorientierte Gestaltung von Lernumgebungen. Weinheim: Beltz.

Lamon, M., Secules, T., Petrosino, A. J., Hackett, R., Bransford, J. D. \& Goldman, S. R. (1996). Schools for Thought: Overview of the project and lessons learned from one of the sites. In L. Schauble \& R. Glaser (Eds.), Innovations in learning. New environments for education (pp. 243-288). Mahwah, NJ: Erlbaum.

Lave, J. (1991). Situating learning in communities of practice. In L. B. Resnick, J. M. Levine \& S. D. Teasley (Eds.), Perspectives on socially shared cognition (pp. 63-82). Washington, D.C.:American Psychological Association.

Lave, J. \& Wenger, E. (1991). Situated learning: Legitimate peripheral participation. New York: Cambridge University Press.

Mandl, H., Gruber, H. \& Renkl, A. (1993). Das träge Wissen. Psychologie heute, 20, (9), 64-49.

Mandl, H., Gruber, H. \& Renkl, A. (1995). Situiertes Lernen in multimedialen Lernumgebungen. In L. J. Issing \& P. Klisma (Hrsg.), Information und Lernen mit Multimedia (S. 167-178). Weinheim: Beltz. 
National Council of Teachers of Mathematics (1989). Curriculum and evaluation standards for school mathematics. Reston, VA: NCTM.

Resnick, L. B. (1987). Learning in and out of school. Educational Researcher, 16, (9), 13-20.

Reusser, K. (1988). Problem solving beyond the logic of things: Contextual effects on understanding and solving word problems, Instructional Science, 17, 309-338.

Rogoff, B. (1990). Apprenticeship in thinking. New York: Oxford University Press.

Scardamaglia, M. \& Bereiter, C. (1985). Fostering the development of self-regulation in children's knowledge processing. In S. F. Chipman, J. W. Segal \& R. Glaser (Eds.), Thinking and learning skills: Research and open questions, Vol 2 (pp. 563-578). Hillsdale, NJ: Erlbaum).

Scardamaglia, M., Bereiter, C. \& Lamon, M. (1994). The CSILE Project: Trying to bring the classroom into world 3. In K. M. Gilly (Ed.), Classroom lessons: Integrating cognitive theory and classroom practice (pp. 201-228). Cambridge, MA: MIT Press/Bradford Books.

Sharp, D. L. M., Bransford, J. D., Goldman, S. R., Risko, V. J., Kinzer, C. K. \& Vye, N. J. (1995). Dynamic visual support for story comprehension and mental model building by young, at-risk children. Educational Technology Research and Development, 43 (4), 25-42

Sherwood, R., Kinzer, C., Hasselbring, T. \& Bransford, J. (1987). Macro-contexts for learning: Initial findings and issues. Journal of Applied Cognition, 1, 93-108.

Simon, H. A. (1980). Problem solving and education. In D. T. Tuma \& R. Reif (Eds.), Problem solving and education: Issues in teaching and research (pp. 81-96). Hillsdale, NJ: Erlbaum.

Tripp, S. D. (1996). Theories, traditions, and situated learning. In H. McLellan (Ed.), Situated learning perspectives (pp. 155-). Englewood Cliffs, NJ: Educational Technology Publications.

von Hentig, H. (1984). Das allmähliche Verschwinden der Wirklichkeit. Ein Pädagoge ermuntert zum Nachdenken über die neuen Medien. München: Hanser.

Vygotsky, L. S. (1978). Mind in society: The development of higher psychological processes. Cambridge, MA: Harvard University Press.

Weise, R. (2001). Expository literacy in first grade: Bridging the transition from narrative to expository tasks and texts. Unpublished doctoral dissertation, Vanderbilt University, Nashville, TN.

Weise, R. \& Scharnhorst, U. (2001b). Learning to research in first grade: Scaffolding children's transition from narrative to expository tasks and texts. Paper presentet at the 9th European Conference for Research on Learning and Instruction (EARLI), Fribourg, Switzerland.

Weise, R. \& Scharnhorst, U. (2001b, August). Making visual the research process: Using an expository learning environment to scaffold children's understanding of research in science and other subjects. Paper presentet at the 9th European Conference for Research on Learning and Instruction (EARLI), Fribourg, Switzerland.

Whitehead, A. N. (1929). The aims of education. New York: MacMillan.

Wood, D., Bruner, J. S. \& Ross, G. (1976). The role of tutoring in problem solving. Journal of Child Psychology and Psychiatry, 17, 89-100. 


\section{Anchored Instruction: Apprentissage contextualisé dans des environnements multimédia}

\section{Résumé}

"Anchored instruction» est une approche constructiviste américaine. Elle est identifiée avec des environnements d'apprentissage assistés par des moyens technologiques qui visent différentes branches et degrés scolaires. Ces environnements se présentent aux élèves sous forme de contextes narratifs motivants et les confrontent à des problèmes complexes à résoudre. Les objectifs envisagés ainsi que les principes suivis dans la construction et l'implémentation de ces environnements d'apprentissage sont illustrés à l'aide de deux exemples d'application: (1) «The Adventures of Jasper Woodbury», une série de narrations présentées sur vidéo qui sont utilisées dans l'enseignement des mathématiques, et (2) «The Little Planet Literacy Series» qui propose aux élèves de première année scolaire différentes activités d'apprentissage en relation avec des narrations fictives sur vidéo afin de les introduire à la lecture et à l'écriture. Quelques résultats de recherche relatifs aux deux applications sont résumés.

Ensuite, une taxonomie est présentée qui montre que l'apprentissage dans ces environnements est soutenu par des combinaisons d'aides au niveau social et technologique. En vue des efforts considérables requis pour le développement et l'implémentation de tels environnements, quelques questions critiques sont discutées à la fin de l'article.

\section{Anchored Instruction: apprendimento in situazione in ambienti di apprendimento multimediali}

\section{Riassunto}

"Anchored Instruction» è un approccio costruttivista americano i cui rappresentanti sviluppano, applicano e verificano piattaformi di apprendimento su basi tecnologiche per materie e per età diverse. L'esposizione di due applicazioni permette di illustrare gli obiettivi, le caratteristiche e i principi generatori di queste piattaforme: si tratta di "The Adventures of Jasper Woodbury», una serie di video-storie con materiali aggiuntivi che confrontano gli allievi con problemi matematici complessi e di "The Little Planet Literacy Series», che introduce principianti alla lettura e alla scrittura attraverso storie animate e attività multimediali. Vengono esposti alcuni risultati emersi dalla ricerca svolta sull'applicazione delle due piattaforme e si mostra tramite una tassonomia come la "Anchored Instruction» sia in grado di combinare forme di supporto sociale e tecnologico in modo da favorire la flessibilità e il transfer di quanto appreso. In conclusione si formulano alcune domande critiche relative agli elevati costi di creazione e implementazione di ambienti di apprendimento del genere. 


\title{
Anchored Instruction: Situated Learning in Multimedia Learning Environments
}

\begin{abstract}
"Anchored Instruction» is an American constructivist approach that is known for the development and implementation of technologically supported learning environments for various school subject matters and age levels. Student learning is situated in age appropriate, complex, and engaging macro-contexts. Social and technological scaffolds are combined to ensure problem solving as well as flexible use and transfer of learned knowledge. Important goals, design principles, and implementation features of these learning environments are illustrated by presenting two applications: (1) "The Adventures of Jasper Woodbury», a series of video-based stories and additional materials exposing students to complex mathematical problems, and (2) «The Little Planet Literacy Series» introducing first graders to reading and writing by linking conventional and multimedia learning activities to video-based fictional stories. Some research findings related to these applications are summarized.

Further, a taxonomy of social and technological learning scaffolds that are typical for anchored instruction environments is presented. Considering the substantial effort required for the development and implementation of the learning environments, a number of critical questions and issues are discussed at the end of the article.

Further, a taxonomy of social and technological learning scaffolds that are typical for anchored instruction environments is presented. Considering the substantial effort required for the development and implementation of the learning environments, a number of critical questions and issues are discussed at the end of the article.
\end{abstract}

